\title{
Frequency and Time-Frequency Analysis of Acoustic Cavitation Noise in Insulating Oils
}

\author{
M. Szmechta*, T. Boczar And P. Frącz \\ Institute of Electric Power Engineering, Opole University of Technology \\ Prószkowska 76, 45-758 Opole, Poland
}

\begin{abstract}
The paper presents some observations of ultrasound induced cavitation phenomenon in a mineral insulating oil. The acoustic cavitation phenomenon was generated at different signal frequencies and rms voltage value supplied to round piezoelectric transducers. Used transducers have created a high energy acoustic field in prepared insulating oil samples. The acoustic emission signal contains a broad-band noise and a fundamental frequency component and its harmonics, produced by the direct driving acoustic field. The paper presents the spectral analysis of acoustic emission signal observed during acoustic cavitation in insulating oils. To emphasize the changes in acoustic cavitation intensity also time-frequency analysis was used and some spectrograms were evaluated. The measurement uncertainty of some frequency-rms voltage pairs was very unfavorable. The main application expected with created cavitation measurement system is to develop a diagnostic tool for the aging of oil, based on the hypothesis that the measured cavitation intensity and spectrum could be affected by aging. Previous published measurements showed some relationship between insulating oil aging process and changes of acoustic emission of cavitations.
\end{abstract}

PACS: 02.60.Lj, 43.40.Le

\section{Acoustic cavitation in insulating oils}

The cavitation phenomenon is a process of rupturing a liquid by decrease in pressure at roughly constant liquid temperature. The behavior of creation of cavitation voids or bubbles in a liquid is a result of increasing liquid flow in pumps or propellers and decreasing liquid pressure on an orifice. An oscillating cavitation bubble can be also created by acoustic field and the phenomenon is called acoustic cavitation $[1,2]$.

Acoustic cavitation is of importance in many technical applications, such as sonochemistry, ultrasonic cleaning, laser surgery in medicine, and lithotripsy. One of the most interesting consequences of cavitation is a sonoluminescence phenomenon in form of multi-bubble sonoluminescence and single-bubble sonoluminescence [3, 4]. An acoustic emission signal from working transformer can be used for monitoring of its condition and recognizing the form of partial discharges generated in insulating oil. That acoustic signal could generate some cavitation bubbles $[5,6]$.

An imploding gas-vapour bubble during cavitation is a source of broad-band acoustic emission signal [7]. A detailed explanation about the acoustic emission by the cavitation bubbles under two distinct cavitation regimes — stable and transient cavitation is given by Leighton [2] and Young [8]. It is generally known that the acoustic cavitation noise spectrum comprises of various fre-

* corresponding author; e-mail: m.szmechta@po.opole.pl quencies related to the fundamental or the driving frequency $f_{S}$. These frequencies are either the subharmonic $\left(f_{S} / 2, f_{S} / 3, f_{S} / 4, \ldots\right)$, the ultraharmonic $\left(n f_{S} / 2\right.$, where $n=2,3,4, \ldots)$ or the harmonic $\left(n f_{S}\right.$, where $n=2$, $3,4, \ldots)$ of the fundamental frequency $f_{S}$. Individual spectral bands of the cavitation radiation are characterized by a finite width and even by definite shape [9]. The acoustic emission signal contains also a broadband noise generated by shock waves of collapsing bubbles of a wide range of sizes [10].

The analysis of hydrodynamic cavitation acoustic emission spectrum can be used for on-line diagnostics of a centrifugal pump in which cavitation is the source of instability of the pump and caused head capacity and efficiency curves $[11,12]$. Cavitation noise were also observed in acoustically driven mineral insulating oil filled vessel [13-17].

The properties of mineral oils used for insulation and cooling in power engineering e.g. power transformers, change in time. Many factors (temperature, humidity, external electrical field) influences the deterioration of the oil [18-20]. Transformer aging is mainly related to the degradation of the insulation, caused mainly by the thermal stress of the insulating paper, together with the electrochemical decomposition of the oil. The physicochemical processes can be very complex [21, 22].

Dissolved gas analysis (DGA) is the most important tool in determining the condition of a transformer. It is the first indicator of a problem and can identify deteriorating insulation and oil, overheating, hot spots, partial discharge, and arcing. The health of the oil is reflec- 
tive of the health of the transformer itself. Dissolved gas analysis consists of sending transformer oil samples to a commercial laboratory for testing and therefore is very expensive [23, 24].

\section{Experimental setup}

This paper presents an experimental study dealing with the spectral analysis of the acoustic emission cavitation phenomenon in mineral oil, carried out with a vessel fitted with piezoelectric transducers to induce acoustic-excited cavitation. A round bottomed glass flask was used as a cavitation vessel. Used transducers created a high energy acoustic field in prepared insulating oil sample.

The used experimental setup consists of four main units (Fig. 1):

- Acoustic cavitation generation unit;

- Acoustic emission measurement unit;

- Microcontroller based central control system communicating with other units;

- MATLAB based communication and data analysis application.

More details about used experimental setup can be found in [24]. The schematic of the measurement device is shown in Fig. 1.

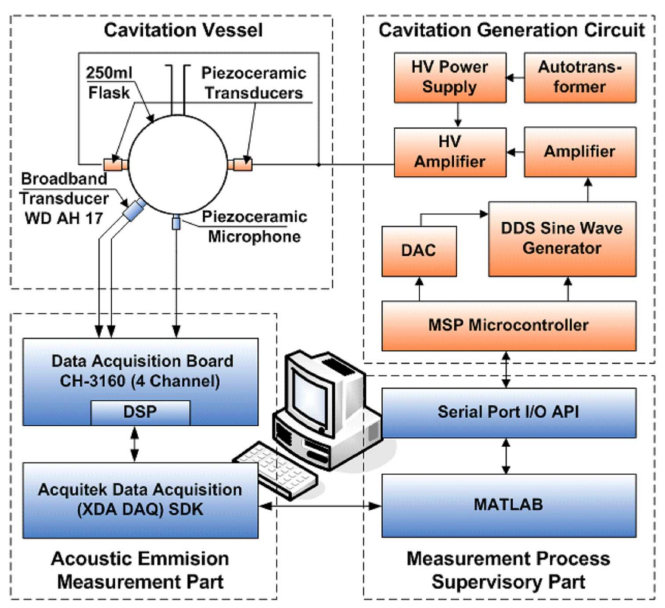

Fig. 1. Schematic of experimental apparatus.

\section{Frequency analysis of acoustic cavitation noise in insulating oils}

Acoustic cavitation known as the formation, growth and rapid collapse of gas-vapor bubbles in surrounding liquid during acoustic irradiation was investigated theoretically and experimentally many times since the first time ultrasonics were used for cavitation excitation. The cavitation bubbles dynamics according to the RayleighPlesset equation and its modified versions proposed by another researchers in the last decades depends mainly on two factors $[2,3,25]$ :

- the parameters of source for generation of acoustic field i.e. its intensity and frequency;

- the properties of used liquid medium and the content of dissolved gases. The various physical properties of the media are represented by its density, surface tension, vapor pressure, polytropic constant, thermal diffusivity and other parameters of the medium.

Aging of transformer in service is due to the combination of different stresses like electrical, magnetic, mechanical and thermal. Decomposition of the oil is a consequence of these stresses. During oil degradation process gases will evolve as the hydrocarbon chains break down leaving large free radicals in the liquid phase. The most significant gases appearing during degradation of transformer mineral oils are $\mathrm{O}_{2}, \mathrm{~N}_{2}, \mathrm{H}_{2}, \mathrm{CH}_{4}, \mathrm{CO}, \mathrm{CO}_{2}$, $\mathrm{C}_{2} \mathrm{H}_{6}, \mathrm{C}_{2} \mathrm{H}_{4}, \mathrm{C}_{2} \mathrm{H}_{2}, \mathrm{C}_{3} \mathrm{H}_{8}$ and other liquid hydrocarbons $[22,24,26,27]$.

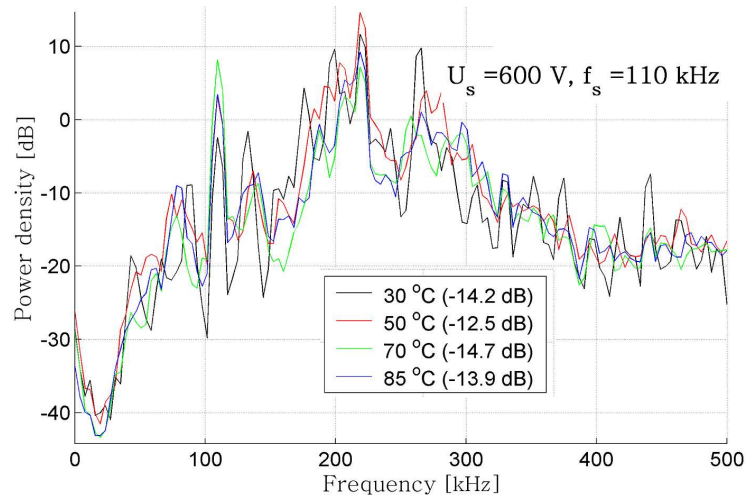

Fig. 2. Power spectral density of several acquired cavitation noise signals at different insulating oil temperatures and its average value in $\mathrm{dB}$.

The paper presents an analysis of acoustic emission signal observed during acoustic cavitation in insulating oils in time and frequency domain. In preview research works several efficiently descriptors of cavitation intensity in frequency domain have been proposed. Power spectral density estimation of a discrete-time signal vector using Welch's averaged, modified periodogram method was used as a data analysis tool in frequency domain. Power spectral density of several acquired cavitation noise signals at different insulating oil temperatures and its average value in $\mathrm{dB}$ are presented in Fig. 2. Then a proposed cavitation intensity factor $P_{\mathrm{CAV}}(1)$ was calculated from power spectral density using the following formula: 


$$
P_{\mathrm{CAV}}=\frac{1}{N_{f}} \sum_{n=1}^{N_{f}} \operatorname{PSD}\left(f_{n}\right),
$$

where $P_{\mathrm{CAV}}$ - cavitation intensity factor; $\operatorname{PSD}\left(f_{n}\right)-$ power density of acoustic emission signal at $n$-th frequency component $\left(f_{n}\right) ; N_{f}$ - the size of used estimated output frequency vector during the estimation of PSD.

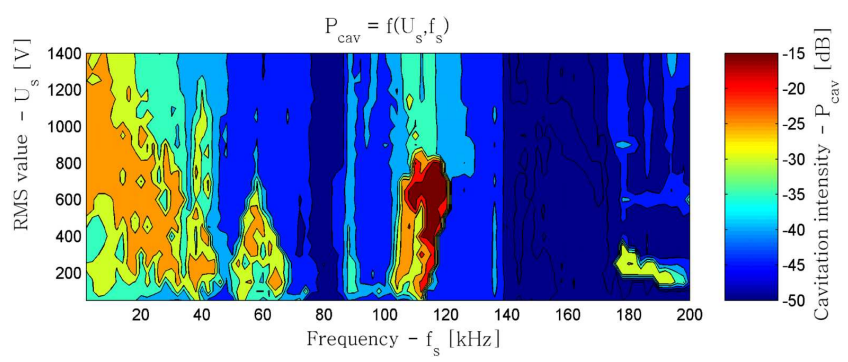

Fig. 3. The results of introduced cavitation intensity factor $P_{\mathrm{CAV}}$.

The round bottomed $250 \mathrm{ml}$ cavitation vessel was filled with fresh mineral oil with $0.022 \mathrm{mgKOH} / \mathrm{g}$ acidicity, $0.88 \mathrm{~kg} / \mathrm{dm}^{3}$ density at $20^{\circ} \mathrm{C}$ and $9.48 \mathrm{~mm}^{2} / \mathrm{s}$ viscosity at $40^{\circ} \mathrm{C}$. The main aim of this work is to point out the possibility of using acoustic emission signal from cavitation induced by a high energy acoustic field as a diagnostic tool for insulating oil aging properties. From the cavitation intensity diagram presented in this paper (Fig. 3) a parallelogram shaped area of signal voltage-frequency pairs can be observed at which a high intensity cavitation can occur. The most intense cavitation was observed at voltages between $400 \mathrm{~V}$ and $800 \mathrm{~V}$ and at frequencies from $107 \mathrm{kHz}$ and $117 \mathrm{kHz}$. The next step in our research will be to find the relationship between the cavitation intensity diagram shape and aging properties of insulating oil samples used as cavitation liquid.

\section{Time-frequency analysis of acoustic cavitation noise in insulating oils}

The second step in carried out acoustic emission signal analysis of generated acoustic cavitation phenomenon in insulating oil was to study the signal in both the time and frequency domains simultaneously. The following two figures show two extreme cases of observed acoustic emission signals. The first one, as depicted in Fig. 4, shows the results of time-frequency analysis of acoustic emission signal sample in case of low cavitation intensity (low $P_{\text {CAV }}$ value). The cavitation phenomenon was generated at source signals frequency $f_{S}=100 \mathrm{kHz}$ and its rms value $U_{S}=200 \mathrm{~V}$. In prepared spectrogram the power spectral density of the signal is constant in time.

The second case, as depicted in Fig. 5, shows the results of time-frequency analysis of acoustic emission signal sample in case of high cavitation intensity (the determined cavitation intensity factor value was $P_{\mathrm{CAV}}=$ $-14.7 \mathrm{~dB}$ ). The cavitation phenomenon was generated

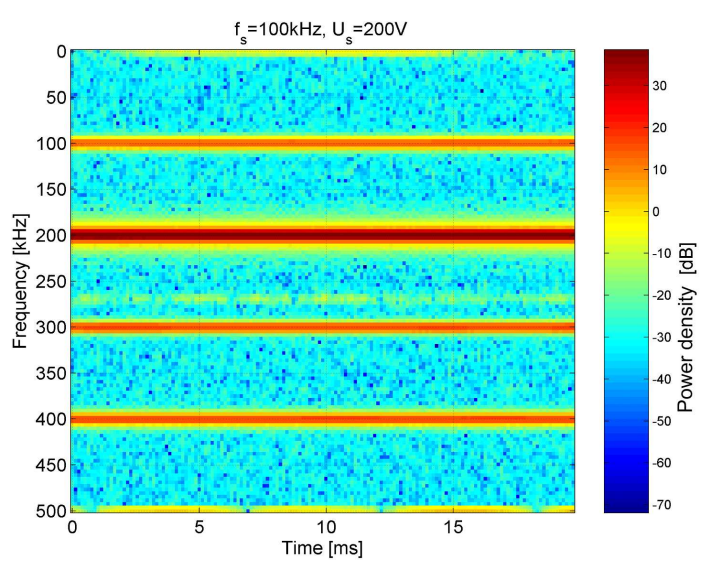

Fig. 4. The results of time-frequency analysis of acoustic emission signal sample in case of low cavitation intensity.

in that case at source signals frequency $f_{S}=115 \mathrm{kHz}$ and its rms value $U_{S}=600 \mathrm{~V}$. In prepared spectrogram the power spectral density of the signal is also constant in time independently of the actual power density.

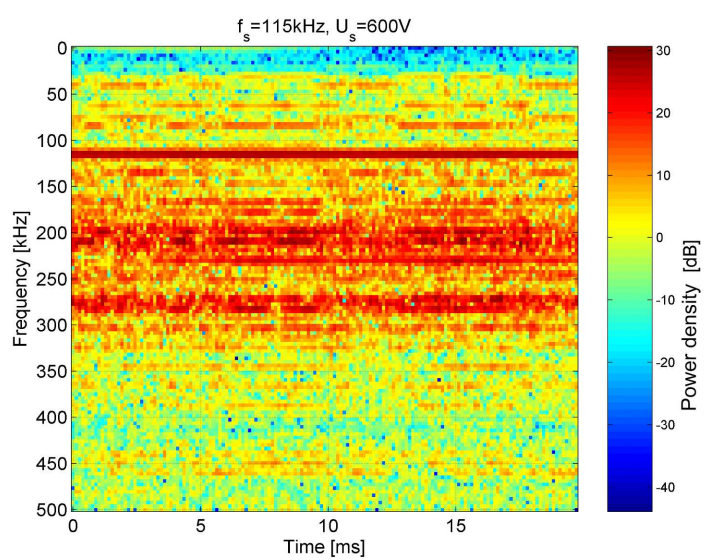

Fig. 5. The results of time-frequency analysis of acoustic emission signal sample in case of high cavitation intensity.

\section{Conclusion}

The subject area taken in this article is a result of a cycle of research work concerning the analysis of ultrasonic cavitation phenomenon in mineral insulating oils. The article includes an analysis of the impact of acoustic cavitation generation unit working point for an acoustic emission signal generated during cavitation phenomenon. This study aims to develop the basic methods of analysis and interpretation of measurement data to estimate the degree of intensity of cavitation. The study focuses on spectral analysis of acoustic emission signal and changes in the spectra of recorded signals. We investigated the acoustic emission of cavitation accompanying the forced 
signal frequencies ranging from $0 \mathrm{kHz}$ to $200 \mathrm{kHz}$ in steps of $2 \mathrm{kHz}$ and rms values range from $0 \mathrm{~V}$ to $1400 \mathrm{~V}$ in steps of $50 \mathrm{~V}$. Used sampling frequency of acoustic emission signal was $1 \mathrm{MHz}$. The thesis placed in the research project talks about the possibility of estimating the degree of aging of insulating oil on the basis of spectral analysis of acoustic emission signals accompanying cavitation phenomenon. In order to validate the hypothesis experimental research will be conducted according to the cavitation intensity factor $P_{\mathrm{CAV}}$ dependence on aging time $t_{\text {st }}$ of insulating oil sample, and a model will be proposed according to the results of measurements. Mapping changes were also made of $P_{\mathrm{CAV}}$ factor ratio depending on the parameters of the source signal used for the generation of acoustic cavitation in the form of two-dimensional color contour plots. These plots allowed us to identify areas most suitable for the acoustic cavitation phenomena in insulating oils. To emphasize the changes in acoustic cavitation intensity also time-frequency analysis was used and some spectrograms were evaluated. In prepared spectrograms the power spectral density of the signal is also constant in time independently of the actual power density.

\section{Acknowledgments}

This work is financed with the means for science as an individual research project no. N N511 351137.

\section{References}

[1] K.S. Suslick, Y. Didenko, M.M. Fang, T. Hyeon, K.J. Kolbeck, W.B. McNamara,. M.M. Mdleleni, M. Wong, Philos. Trans. R. Soc. Lond. A $\mathbf{3 5 7}$, 335(1999).

[2] T.G. Leighton, The Acoustic Bubble, Academic Press, London 1994.

[3] B. Dey, S. Aubry, Physica D 12, 216 (2006).

[4] K. Yasui, Phys. Rev. E 60, 1759 (1999).

[5] T. Boczar, S. Borucki, A. Cichoń, M. Lorenc, Mol. Quant. Acoust. 26, 35 (2005).

[6] T. Boczar, IEEE Trans. Diel. Electr. Ins. 8, 598 (2005)
[7] S. Hayashi, Y. Kato, K. Tanaka, H. Kobayashi, Acoust. Sci Technol. 22, 221 (2001).

[8] F.R. Young, Cavitation, McGraw-Hill, London 1989.

[9] A.O. Maksimov, E.V. Sosedko, WCU 2003, Paris, 2003, p. 593.

[10] M. Hodnett, R. Chow, B. Zeqiri, Ultrason. Sonochem. 11, 47 (2004).

[11] L. Alfayez, D. Mba, G. Dyson, NDT $\&$ E International 38, 354 (2005).

[12] W. Stanisławski, M. Zmarzły, M. Szmechta, Przeglad Elektrotechniczny 3, 144 (2008) (in Polish).

[13] D. Zmarzły, M. Szmechta, Pomiary Automatyka Kontrola 54, 64 (2008) (in Polish).

[14] M. Szmechta, D. Zmarzły, M. Lorenc, Acta Phys. Pol. A 114, A231 (2008).

[15] M. Szmechta, D. Zmarzły, T. Boczar, M. Lorenc, Europ. Phys. J.-Spec. Top. 154, 211 (2008).

[16] T. Boczar, M. Szmechta, D. Zmarzły, in: IEEE ICDL Poitiers-France, ECS Groupe 2008, p. 1.

[17] M. Szmechta, T. Boczar, D. Zmarzły, P. Aksamit, in: ISEI 2010, San Diego (USA) 2010, p. 137.

[18] T. Boczar, D. Zmarzły, IEEE Trans. Diel. Electr. Ins. 13, 632 (2006).

[19] PN-EN 60296 (2007).

[20] T. Pustelny, I. Zielonka, C. Tyszkiewicz, P. Karasiński, B. Pustelny, Opto-Electron. Rev. 14, 161 (2006).

[21] J. Kędzia, IEEE EI 24, 197 (1989).

[22] R. Malewski, Transformers in Exploatation, Energo-Complex, Chorzów 2005 (in Polish).

[23] C.T. Dervos, C.D. Paraskevas, P.D. Skafidas, N. Stefanou, in: IEEE ICDL 2005, EPIROB, Coimbra 2005, p. 233.

[24] I. Fist, Transformer Diagnostics, Wiley, London 2003.

[25] M. Szmechta, Ph.D. Thesis, Opole University of Technology, 2009.

[26] T. Saha, IEEE Trans. Dielectr. Electr. Ins. 10, 903 (2003).

[27] P. Struk, T. Pustelny, K. Gut, K. Gołaszewska, E. Kamińska, M. Ekielski, I. Pasternak, E. Łusakowska, A. Piotrowska, Acta Phys.Pol. A 116, 414 (2009). 